\title{
PATTERN SELECTION IN THREE DIMENSIONAL DENDRITIC GROWTH
}

\author{
D. A. KESSLER ${ }^{1}$ and H. LEVINE ${ }^{2}$ \\ 'Department of Physics, University of Michigan, Ann Arbor, MI 48109 and 2 Department of Physics, \\ B-019, University of California at San Diego, La Jolla, CA 92093, U.S.A.
}

(Received 25 March 1987; in revised form 9 March 1988)

\begin{abstract}
We study the selection of the shape and growth velocity of three dimensional dendritic crystals in cubically anisotropic materials. We demonstrate that aside from minor additional complexities due to the lack of axisymmetry, the recently discovered mechanism of "microscopic solvability" can be extended to these systems and used to find a unique needle crystal solution of the equations of thermal diffusion-controlled solidification. We compare the predictions of this approach with measured growth rates in succinonitrile. Finally, we extend our analysis to determine the ratio of the sidebranch wavelength to the tip radius.
\end{abstract}

Résumé-Nous étudions la sélection de forme et de vitesse de croissance de cristaux dendritiques tridimensionnels dans des matériaux cubiques anisotropes. Nous montrons que, mises à part quelques difficultés supplémentaires mineures dues au manque de symétrie axiale, le mécanisme dit de "résolubilité microscopique", récemment découvert, peut être étendu à ces systèmes et utilisé pour trouver une solution unique de cristal en aiguille pour les équations de la solidification thermique régie par la diffusion. Nous comparnns les prévisions de cette approche avec les vitesses de croissance expérimentales dans le succinonitrile. Enfin, nous élargissons notre analyse pour déterminer le rapport de la longueur des branches latérales au rayon de l'extrémité de la dendrite.

\begin{abstract}
Zusammenfassung-Wir behandeln die Auswahl von Form und Wachstumsrate dreidimensionaler dendritischer Kristalle in kubisch anisotropen Materialen. Wir zeigen, daß-abgesehen von kleineren zusätzlichen Verkomplizierungen durch die fehlende Axisymmetrie- der kürzlich aufgefundene Mechanismus der "mikroskopischen Löslichkeit" auf diese Systeme ausgedehnt werden kann; damit kann eine einheitliche Nadelkristall-Lösung der Gleichungen für die durch thermische Diffusion gesteuerte Erstarrung erhalten werden. Die Aussagen dieser Näherung vergleichen wir mit den an Succinonitril gemessenen Werten. Zum SchluB erweitern wir unsere Analyse, um das Verhältnis der Wellenlänge der Seitenarme zum Spitzenradius zu ermitteln.
\end{abstract}

\section{INTRODUCTION}

Recently, the problem of velocity selection for twodimensional dendritic crystal growth [1] was solved both numerically $[2,3]$ and analytically $[4-7]$. The solution relies upon a solvability mechanism present when one tries to construct steady-state solutions of the interface evolution equation in the presence of non-zero surface tension. We have referred to this concept as the "microscopic solvability" approach. The predictions of this analysis agree with this numerical simulations of Saito et al. [8] and are qualitatively consistent with all of what is known about true dendritic crystals. A quantitative comparison has been lacking to the simple fact that most experiments are three dimensional in nature, whereas the theory has been restricted to two.

The problem of three dimensional dendrites is difficult to solve exactly, because the existence of steady-state solutions (parabolic needle crystals) requires non-isotropic surface tension [9-11]. Any physical anistropy (such as that arising, say, from an underlying cubic symmetry) will give rise to a nonaxisymmetric form for the shape. This fact greatly increases the complexity of either the numerical treatment or of any attempted analytic approach.

In this paper, we solve this problem by making use of two approximations. First, we demonstrate numerically that it is possible to linearize the shape around the Ivantsov solution. The validity of this type of approximation has already been verified in two dimensions by Ben-Amar and Moussallam [12]. We perform this demonstration within the context of an axisymmetric anisotropy. Next, we generalize the linearized version to include the effects of nonaxisymmetry. Specifically, we express the interface via a mode expansion in the azimuthal coordinate $\phi$

$$
z(r, \phi)=-\frac{r^{2}}{2 p}+\sum_{m=0}^{M-1} \delta_{m}(r) \cos 4 m \phi .
$$

In the above expression, $p$ is the Peclet number and the interface is assumed to be moving in the $\hat{z}$ direction.

Clearly, the above truncation cannot be an exact solution. Nevertheless, we will show that this approach can yield quantitatively accurate results. Note that we have in mind $M \sim 2,3$; if $M$ is chosen to 
be large (100 or so), the approximation for the azimuthal dependence of $z$ would be as accurate as that used for the $r$ dependence.

The introduction of non-axisymmetric shapes for the steady-state solution leads to an interesting puzzle which must be resolved by the solvability approach. The standard methodology of looking for a steadystate solution by relaxing the condition $\partial z / \partial r=0$ near the tip leads to a set of $M$ independent constraints, one for each $\delta_{m}$ in equation (1). We must therefore be able to vary $M$ parameters so as to find a smooth solution. Suprisingly, we find that the asymptotic shape of the non-axisymmetric needle crystal contains precisely enough degrees of freedom to accomplish this task. In practice, the axisymmetric condition $\delta_{0}^{\prime}(0)$ is most sensitive to the dimensionless velocity and the remaining conditions are important only for internal consistency. We will see this in detail in Section 5.

The outline of this work is as follows. In the following Section, we review the derivation of the integro-differential equation governing the interface evolution. The only part of this which is technically difficult is the three dimensional generalization of the Gibbs-Thomson condition for anisotropic surface energy. In Section 3, we restrict ourselves to the axisymmetric limit and derive the selected velocity. In Section 4, we describe the linearization and verify that the results for the axisymmetric case agree with those of the preceding non-linear method. In Section 5 , we solve a two mode truncation and argue that this should provide an accurate prediction for the full problem. Applying this reasoning, we compute the selected velocity of succinonitrile and compare our results to the data of Huang and Glicksman [13]. In Section 6 , we derive the peak sidebranching wavelength following the method of Ref. [14]. The final section summarizes our results and describes the still puzzling case of pivalic acid crystals.

\section{STEADY-STATE EQUATION}

We start our analysis with the usual assumptions [15] regarding a crystal immersed in a supersaturated melt and whose growth is controlled by thermal diffusion. The experimental control parameter is the dimensionless undercooling

$$
\Delta=\frac{T_{\mathrm{m}}-T_{\infty}}{L / c_{\mathrm{p}}}
$$

where $T_{\mathrm{m}}$ is the melting temperature, $L$ the latent heat and $c_{p}$ the specific heat. The surface energy $\bar{\gamma}$ is assumed to arise from an underlying cubic crystal. We measure this surface energy in dimensionless units by making use of an assumed velocity of motion. This leads us to the expression

$$
\begin{aligned}
& \gamma(\tilde{\theta}, \tilde{\phi}) \equiv \frac{c_{\mathrm{p}} \bar{\gamma} T_{\mathrm{m}} v}{2 L^{2} D}=\gamma_{0}[1+4 \mathrm{eg}(\tilde{\theta}, \tilde{\phi})] \\
& \quad g(\tilde{\theta}, \tilde{\phi})=\cos ^{4} \tilde{\theta}+\sin ^{4} \tilde{\theta}\left(\cos ^{4} \tilde{\phi}+\sin ^{4} \tilde{\phi}\right)
\end{aligned}
$$

where $\tilde{\theta}, \tilde{\phi}$ are the spherical angles made by the normal vector to the interface and $D$ is the thermal diffusivity. The parameter $\epsilon$ measures the strength of the anisotropy, giving a maximum of surface energy in the (100) crystal direction.

The evolution equation can be found using standard techniques. The temperature field is taken to satisfy the diffusion equation with the interface acting as a source of magnitude $t_{\mathrm{n}} L / c_{\mathrm{p}}$ for normal velocity $v_{\mathrm{n}}$. This leads to the general evolution equation

$$
\begin{aligned}
\Delta-\Delta_{\mu}= & \int_{0}^{\infty} \frac{\mathrm{d} \tau}{(4 \pi D \tau)^{3 / 2}} \int \mathrm{d}^{2} s^{\prime} v_{\mathrm{n}}\left(r^{\prime}, \phi^{\prime}, t-\tau\right) \\
& \times \exp \left[\frac{\left.\begin{array}{c}
-\left(r^{2}+r^{\prime 2}-2 r r^{\prime} \cos \left(\phi-\phi^{\prime}\right)\right. \\
\left.+\left[z(r, \phi, t)-z\left(r^{\prime}, \phi^{\prime}, t-\tau\right)\right]^{2}\right)
\end{array}\right]}{4 D \tau}\right]
\end{aligned}
$$

where $\Delta_{\mu}$ is the Gibbs-Thomson shift in the equilibrium melting point, which we will soon compute. In this expression the interface is given by a function $z(r, \phi, t)$ and $\mathrm{d}^{2} s^{\prime}$ is the surface area measure.

We can simplify the above expression for the case of steady-state motion that will be the primary focus of this work. In this case, $v_{\mathrm{n}}=v \hat{n} \cdot \hat{z}$; the second factor can be used to rewrite the measure using

$$
\mathrm{d}^{2} s^{\prime} \hat{n}^{\prime} \cdot \hat{z}=r^{\prime} \mathrm{d} r^{\prime} \mathrm{d} \phi^{\prime}
$$

Finally, the integral over $\tau$ can be explicitly performed using $z(r, \phi, t)=z(r, \phi)+v t$. The steadystate equation then takes the familiar form

$$
\Delta-\Delta_{\mu}=\int_{0}^{2 \pi} \frac{\mathrm{d} \phi^{\prime}}{2 \pi} \int_{0}^{\infty} r^{\prime} \mathrm{d} r^{\prime} \frac{\mathrm{e}^{z\left(r^{\prime}, \phi^{\prime}\right)-z(r, \phi)-d}}{d}
$$

where

$$
d=\sqrt{\begin{array}{r}
r^{2}+r^{\prime 2}-2 r r^{\prime} \cos \left(\phi-\phi^{\prime}\right) \\
+\left[z(r, \phi)-z\left(r^{\prime}, \phi^{\prime}\right)\right]^{2}
\end{array}} .
$$

In the above expressions, all lengths have been rescaled by the diffusion length $v / 2 D$.

It is possible to verify [16] that Ivantsov [17] paraboloid of revolution $z=-r^{2} / 2 p$ satisfies equation (4) if the Gibbs-Thomson shift is set equal to zero and the Peclet number $p$ is related to the undercooling by the three dimensional Ivantsov formula

$$
\Delta=p e^{p} E_{1}(p)
$$

where $E_{1}$ is the exponential integral function. We will use this solution later as the starting point for our linearization.

We now turn to an evaluation of $\Delta_{\mu}$, the melting point depression due to interfacial curvature. To do this, we consider a shifted surface (parameterized by cartesian coordinates) to first order in the shift

$$
z(x, y)=z_{0}(x, y)+\psi(x, y) .
$$

Let us assume that the surface energy is given by a function $\gamma$ which depends on surface orientation. The 
surface energy is then given by

$$
S=\int \mathrm{d} x \mathrm{~d} y \gamma(\tilde{\theta}, \tilde{\phi}) \sqrt{1+(\vec{\nabla} z)^{2}}
$$

where the angles $\tilde{\theta}, \tilde{\phi}$ are the actual spherical angles made by the interface normal and $\vec{\nabla}$ is the two dimensional gradient operator. Under the interface shift, the change in surface energy is therefore

$$
\begin{aligned}
\delta S=\int \mathrm{d} x \mathrm{~d} y\left[\frac{1}{\sqrt{1+(\vec{\nabla} z)^{2}}} \vec{\nabla} z \cdot \vec{\nabla} \psi \gamma(\tilde{\theta}, \tilde{\phi})\right. \\
\left.+\sqrt{1+(\vec{\nabla} z)^{2}}\left(\delta \tilde{\theta} \frac{\partial \gamma}{\partial \tilde{\theta}}+\delta \tilde{\phi} \frac{\partial \gamma}{\partial \tilde{\phi}}\right)\right]
\end{aligned}
$$

Since $\tilde{\theta}$ equals $\tan ^{-1}|\vec{\nabla} z|$, we have

$$
\delta \bar{\theta}=\frac{\vec{\nabla} z \cdot \vec{\nabla} \psi}{\left(1+(\vec{\nabla} z)^{2}\right)|\vec{\nabla} z|}
$$

and similarly

$$
\delta \tilde{\phi}=\frac{-\hat{z} \cdot(\vec{\nabla} \psi \times \vec{\nabla} z)}{|\vec{\nabla} z|^{2}}
$$

Integrating by parts allows us to remove all the derivatives acting on the shift $\psi$ and rewrite this expression as the integral of $\psi$ multiplied by some prefactor. Finally we note that this prefactor is exactly the Gibbs-Thomson shift $\Delta_{\mu}$; this is because the equilibrium condition requires that the surface energy term be exactly compensated for by a change in volume free energy

$$
\delta F=\int \mathrm{d} x \mathrm{~d} y \psi(x, y) \Delta_{\mu}(x, y) .
$$

After some tedious algebra, we arrive at the final expression

$$
\begin{aligned}
& -\Delta_{\mu}=\gamma \vec{\nabla} \cdot\left(\frac{\vec{\nabla} z}{\sqrt{1+(\vec{\nabla} z)^{2}}}\right) \\
& +\frac{\partial^{2} \gamma}{\partial \vec{\theta}^{2}} \frac{\vec{\nabla} \tilde{\theta} \cdot \vec{\nabla} z}{\sqrt{1+(\vec{\nabla} z)^{2} \mid}|\vec{\nabla} z|} \\
& +\frac{\hat{c}^{2} \gamma}{\partial \hat{\phi} \partial \hat{\theta}} \frac{\vec{\nabla} \tilde{\phi} \cdot \vec{\nabla} z}{\sqrt{1+(\vec{\nabla} z)^{2}}|\vec{\nabla} z|} \\
& -\frac{\partial^{2} \gamma}{\partial \tilde{\phi}^{2}} \frac{(\vec{\nabla} \tilde{\phi} \times \vec{\nabla} z) \cdot \hat{z}}{|\vec{\nabla} z|^{2}} \sqrt{1+(\vec{\nabla} z)^{2}} \\
& -\frac{\partial^{2} y}{\partial \partial \partial \tilde{\phi}} \frac{(\vec{\nabla} 0 \times \vec{\nabla} z) \cdot \approx}{|\vec{\nabla} z|^{2}} \sqrt{1+(\vec{\nabla} z)^{2}} \\
& +\frac{\partial \gamma}{\partial \theta} \frac{\vec{\nabla} \cdot(\vec{\nabla} z /|\vec{\nabla} z|)}{\sqrt{1+(\vec{\nabla} z)^{2}}} \\
& -\frac{\partial \gamma}{\partial \tilde{\phi}} \frac{z \cdot \vec{\nabla} z \times \vec{\nabla}\left(1 /|\vec{\nabla} z|^{2}\right)}{\sqrt{1+(\vec{\nabla} z)^{2}}}
\end{aligned}
$$

This expression reduces to the more standard form if we change variables to consider angles along the principal axes along the interface [16], but the above equation is actually much easier to evaluate given any surface. Note that for isotropic surface tension, we recover $\Delta_{\mu}=\gamma \kappa$ and for two dimensional systems,

$$
\Delta_{\mu}=\left(\gamma+\frac{\partial^{2} \gamma}{\partial \partial^{2}}\right) \kappa
$$

with interface curvature $\kappa$, in accordance with known results.

Equation (10) completes our derivation of the steady-state interface evolution equation. In the next several sections we describe our methodology for solving this equation so as to derive the velocity and shape of the growing dendrite.

\section{THE AXISYMMETRIC LIMIT}

In this section, we study an axisymmetric approximation to the full three dimensional dendritic growth equation. Let us rewrite equation (2) for the surface energy anisotropy as

$$
\begin{aligned}
\gamma(\tilde{\theta}, \tilde{\phi})=\gamma_{0}\left[1+4 \epsilon\left(\cos ^{4} \tilde{\theta}+3 / 4 \sin ^{4} \tilde{\theta}\right.\right. \\
\left.\left.+1 / 4 \sin ^{4} \tilde{\theta} \cos 4 \tilde{\phi}\right)\right] .
\end{aligned}
$$

This expression allows us to define an axisymmetric problem by dropping the $\cos 4 \bar{\phi}$ term in $\gamma$. Substituting this reduced form into equation (10) gives us the axisymmetric Gibbs-Thomson shift

$$
\begin{aligned}
\Delta_{p}=\left(\gamma+\frac{\partial^{2} \gamma}{\partial \theta^{2}}\right) & \frac{-z^{\prime \prime}}{\left(1+z^{\prime 2}\right)^{3 / 2}} \\
& +\left(\gamma+\cot \theta \frac{\partial \gamma}{\partial \theta}\right) \frac{-z^{\prime}}{r\left(1+z^{\prime 2}\right)^{1 / 2}}
\end{aligned}
$$

where ' is $\partial / \partial r$, and we have dropped the "tilde" from the definition $\theta=\tan ^{-1}\left(-z^{\prime}\right)$.

Our goal is to solve equation (4) for $z(r)$. This can be done in the by now standard fashion by first looking for solutions with non-vanishing slope at the tip and then varying $\gamma_{0}$ to satisfy the additional "solvability" condition [19]. We discretize the problem by defining the point

$$
r_{i}=p \tan q_{i} . \quad q_{i}=\frac{i \pi}{2 N}
$$

where $i$ ranges from 0 to $N-1$. The solution should asymptotically approach the Ivanstov paraboloid with a calculable correction derived in Ref. [9]. We therefore set

$$
z\left(r_{i}\right)=-r_{i}^{2} / 2 p+b r_{i}+c \log \left(1+r_{i}\right)+z_{i}
$$

where the coefficients are discussed in Appendix $D$. The unknown variables are now the $z_{i}$. The derivatives on the left hand side of the evolution equation can be computed by simple finite difference techniques. The only slight complication is the fact that there is potentially a singularity in the second term of equation (12) if $z^{\prime}(0) \neq 0$; this is handled by replacing $z^{\prime}(r)$ by the subtracted form $z^{\prime}(r)-z^{\prime}(0)$. Since any physical solution will have $z^{\prime}(0)=0$, this 
modification will not change the allowed shapes and velocities.

The right hand side of the equation was given in general form by equation (4). Since $z$ does not depend on the angular variabie $\phi$, we can evaluate this side of the equation by first computing

$$
\int_{0}^{2 \pi} \frac{\mathrm{d} \phi^{\prime}}{2 \pi} \frac{\mathrm{e}^{-\sqrt{r^{2}+r^{\prime 2}-2 r r^{\prime} \cos \left(\phi-\phi^{\prime}\right)+(\Delta z)^{2}}}}{\sqrt{r^{2}+r^{\prime 2}-2 r r^{\prime} \cos \left(\phi-\phi^{\prime}\right)+(\Delta z)^{2}}} .
$$

The integrand has an integrable singularity and must be handled with care. We write this expression in the subtracted form

$$
\begin{aligned}
& \int_{0}^{2 \pi} \frac{\mathrm{d} \phi^{\prime}}{2 \pi} \frac{\mathrm{e}^{-\sqrt{r^{2}+r^{\prime 2}-2 r r^{\prime} \cos (\phi-\phi)+(\Delta z)^{2}}}-1}{\sqrt{r^{2}+r^{\prime 2}-2 r r^{\prime} \cos \left(\phi-\phi^{\prime}\right)+(\Delta z)^{2}}} \\
& +\int_{0}^{2 \pi} \frac{\mathrm{d} \phi^{\prime}}{2 \pi} \frac{1}{\sqrt{r^{2}+r^{\prime 2}-2 r r^{\prime} \cos \left(\phi-\phi^{\prime}\right)+(\Delta z)^{2}}} .
\end{aligned}
$$

The first integral is always finite and can hence be evaluated by the trapezoidal rule. The second term can be explicitly evaluated in terms of a complete elliptic function

$$
\frac{2}{\pi \sqrt{\left(r+r^{\prime}\right)^{2}+(\Delta z)^{2}}} K\left(\frac{4 r r^{\prime}}{\left(r+r^{\prime}\right)^{2}+(\Delta z)^{2}}\right) .
$$

We next need to evaluate the integral over $r^{\prime}$. Again the integrand contains an integrable singularity, arising from the logarithmic divergence of the elliptic function as its argument approaches 1. Again we make use of a subtraction; in particular, we already know that the Ivantsov solution $z_{0}=-r^{2} / 2 p$ obeys the equation

$$
\Delta=\int_{0}^{2 \pi} \frac{\mathrm{d} \phi^{\prime}}{2 \pi} \int_{0}^{\infty} r^{\prime} \mathrm{d} r^{\prime} \frac{e^{z_{0}\left(r^{\prime}\right)-z_{0}(r)-d}}{d} .
$$

Subtracting this from equation (12) allows us to replace evaluation of the integrand by evaluation of the change in the integrand due to the difference between the actual shape $z(r)$ and the Ivantsov parabola [16]. The resulting integrand is always finite, and hence the resulting $r^{\prime}$ integral can be computed with the trapezoidal rule.

It has already been established [9-11] that there are no steady-state solutions at all for $\epsilon=0$ and that furthermore, the solutions should obey the scaling $\gamma_{0} \sim c^{74}$ for small $\epsilon$. These facts follow immediately from the analogous results in two dimensions and the recognition that the singularity in the three dimensional axisymmetric integral operator is the same as that of the two dimensional one and that furthermore the highest derivative piece in the Gibbs-Thomson shift, $z^{\prime \prime} /\left(1+z^{\prime 2}\right)^{3 / 2}$, again mimics the two dimensional curvature. As we shall soon see, these results are in agreement with the numerical calculations and can serve as an independent means for verifying the accuracy of the results we present.

Our main purpose in this paper is to provide a framework for the eventual quantitative test of this theory of velocity selection. A crucial component of

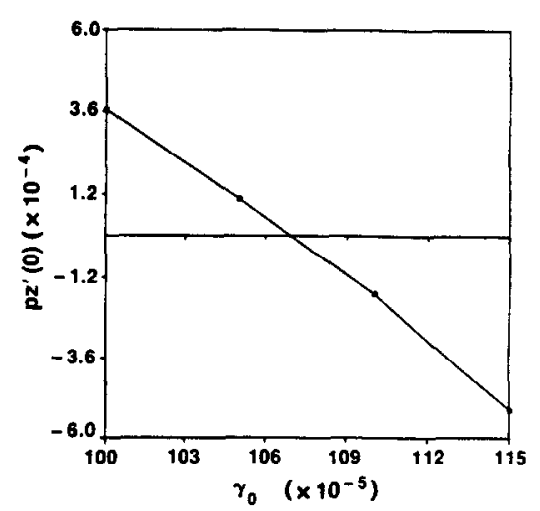

Fig. 1. Slope at tip vs $\gamma_{0} ; N=100, p=0.25$, nonlinear axisymmetric version.

this comparison is the requirement that we accurately know the value of $\epsilon$ in equation (11). In Appendix A, we review the proof [20] that the anisotropy in the equilibrium crystal shape can be used directly to determine $\epsilon$. In the original experimental work, this number was quoted as about $1 \%$ [21]; later work [22] indicated a lowering of this value to $1 / 2 \%$. In this paper we will mostly quote results for the earlier value, recognizing that a re-measurement (perhaps by using digital imaging techniques [23]) would be most valuable.

We now proceed to solve the discretized version of the steady-state equation by Newton's algorithm and thereby compute $z^{\prime}(0)$. Figure 1 represents a typical plot of this mismatch function vs $\gamma_{0}$, at $p=0.25$, for $\epsilon=0.01$. There is a clear zero of this function around $\gamma_{0}=0.00107$. It is easy to check that there are no other solutions at larger values of $\gamma_{0}$. There are additional solutions at smaller values, but in accord with the results [24] in two dimensions, these other shapes are expected to be linearly unstable and hence unphysical.

In Table 1 we present some of our data for several different discretization numbers $N$ and for different Peclet numbers. Note that to this accuracy there is no dependence on the discretization parameter $N$ and accurate results can easily be obtained by extrapolation.

Most importantly, we can verify that at small Peclet numbers, the selected value of $\gamma_{0}$ equals $\sigma^{*} p^{2}$ to high accuracy. This scaling was rigorously derived by Pelce and Pomeau [16] and also follows form previous theories of dendrite shape (such as the marginal stability hypothesis [25]) now known to be

Table 1. Axisymmetric selected $\gamma_{0}$

\begin{tabular}{lrll}
\hline \multicolumn{1}{c}{$p$} & \multicolumn{1}{c}{$N$} & \multicolumn{1}{c}{$\gamma_{0}^{*}$} & $\gamma_{0}^{*} / p^{2}$ \\
\hline 0.05 & 75 & 0.000048 & 0.0194 \\
0.1 & 50 & 0.000188 & 0.0189 \\
0.1 & 75 & 0.000188 & 0.0189 \\
0.25 & 50 & 0.00107 & 0.0171 \\
0.25 & 75 & 0.00107 & 0.0171 \\
0.25 & 100 & 0.00107 & 0.0171 \\
0.4 & 50 & 0.00253 & 0.0158 \\
\hline
\end{tabular}


incorrect. Its origin is that the diffusion length goes to infinity as the Peclet number is lowered and the only relevant macroscopic length scale is the tip radius. Our best estimate for $\sigma^{*}$ is $0.02 \pm 0.002$. It is also worth noting that the trend of $\sigma^{*}$ systematically decreasing as a function of increasing Peclet number also agrees with the experimental results. We will later verify that these trends are not modified upon the inclusion of the effects of non-axisymmetry.

We also have studied the selection at differing values of the anisotropy. For example, at $\epsilon=0.005$, $\sigma^{*}$ is reduced to 0.0079 . This is not quite an $\epsilon^{7 / 4}$ law, which apparently sets in only at smaller $\epsilon$. This type of variation does represent a strong dependence on crystal anisotropy, as expected from previous work on local models and in two dimensions. We will return to this point in the concluding chapter where we return to a discussion of the experiments on succinonitrile and pivalic acid. Now, however, we turn to a method which incorprates nonaxisymmetric terms. This will allow us to show that the above estimate for $\sigma^{*}$ is indeed quantitatively valid for the actual physical system.

\section{LINEARIZATION}

For an exact treatment of the selection problem, it is obviously necessary to generalize the results of Section 3 to arbitrary three-dimensional shapes; i.e. to arbitrary functions $z(r, \phi)$. We will not attempt to carry out such a computation here. Instead, we will introduce two approximations, linearization and mode truncation, which will greatly simplify the computational task. We certainly would not expect these approximations to be qualitatively important; this is due to the fact that the essential mechanism of pattern selection, that of generating the solvability condition, is already present in the simplified problem. It also turns out that these approximations give results that are also quantitatively valid, at the accuracy level at which the experimental data exist. Hence we will be able to make testable predictions for the growth rate $\sigma^{*}$ and the exact shape of the dendrite tip.

Our approach is simply to write

$$
z(r, \phi)=\frac{-r^{2}}{2 p}+\sum_{m=0}^{M-1} \delta_{m}(r) \cos 4 m \phi
$$

linearize in the $\delta_{m}$ and truncate all the Fourier components higher than $4(M-1)$. The resulting equation will then be discretized by the same techniques as used in the last section. This leads to a $M \times N$ set of linear equations which can easily be solved. Again the crucial criterion is that $z^{\prime}(0, \phi)$ must be set equal to zero by proper choice of the selected parameter $\gamma_{0}$. Actually, we will find that the single solvability condition is replaced by $M$ independent conditions, one for each Fourier component of $z$; nevertheless, in each case there turns out to be enough degrees of freedom in the shape to exactly satisfy all of these conditions if and only if $\gamma_{0}$ is precisely chosen.

Let us sketch the derivation of the set of equations we will be using, leaving many of the details to Appendix B. Let us first focus on the right hand side of the steady-state equation, the part containing the integral operator. Upon expanding in $\delta$, we must evaluate

$$
\begin{aligned}
\int_{0}^{2 \pi} \frac{\mathrm{d} \phi^{\prime}}{2 \pi} \int_{0}^{\infty} r^{\prime} \mathrm{d} r^{\prime} \frac{\mathrm{e}^{z_{0}\left(r^{\prime}, \phi^{\prime}\right)-z_{0}(r \cdot \phi)-d}}{d} \\
\times\left[\delta_{m}\left(r^{\prime}\right) \cos 4 m \phi^{\prime}-\delta_{m}(r) \cos 4 m \phi\right] \\
\times\left[1-\frac{z_{0}\left(r^{\prime}\right)-z_{0}(r)}{d}\left(1+\frac{1}{d}\right)\right]
\end{aligned}
$$

with $z_{0}$ again given by the Ivantsov solution and the distance $d$ now computed with $\Delta z \equiv\left[z_{0}(r)-z_{0}\left(r^{\prime}\right)\right]$

$$
d=\sqrt{\left(r+r^{\prime}\right)^{2}-4 r r^{\prime} \sin ^{2}\left(\frac{\phi-\phi^{\prime}}{2}\right)+(\Delta z)^{2}} \text {. }
$$

In expression (15), the integrand contains integrable singularities, which again means that we must be careful in devising a numerical scheme to evaluate this expression. As in Section 3, we deal with this problem via subtraction. For example, consider doing the angular integral

$$
\int_{0}^{2 \pi} \frac{\mathrm{d} \phi^{\prime}}{2 \pi} \frac{\mathrm{e}^{-d}}{d} \cos 4 m \phi^{\prime}
$$

This can be rewritten as

$$
\begin{aligned}
& \int_{0}^{2 \pi} \frac{\mathrm{d} \phi^{\prime}}{2 \pi} \frac{f\left(r, r^{\prime}\right)}{d} \cos 4 m \phi^{\prime} \\
& \quad+\int_{0}^{2 \pi} \frac{\mathrm{d} \phi^{\prime}}{2 \pi} \frac{\mathrm{e}^{-d}-f\left(r, r^{\prime}\right)}{d} \cos 4 m \phi^{\prime}
\end{aligned}
$$

where $f$ is any function which has the property that as we approach the singularity at $r \sim r^{\prime}$, $f \sim 1+O\left[\left(r-r^{\prime}\right)\right]$. The second integral contains no singular points and is therefore simple to evaluate by the trapezoidal rule. The first integral can be done exactly in terms of an elliptic function; the final formula is given in the Appendix. It is convenient to choose $f=\mathrm{e}^{-d_{0}^{3}}$, with $d_{0} \equiv d\left(\phi^{\prime}=\phi+\pi\right)$ so as to make the subtraction vanish rapidly as we move away from $r=r^{\prime}$.

This method can be used for all the terms in the integral operator. For each additional power of $d$ in the denominator of the integrand, we must perform a one higher order subtraction

$$
\frac{\mathrm{c}^{-d}}{d^{2}} \rightarrow \frac{\mathrm{c}^{-d}-\mathrm{e}^{-d_{0}^{3}}(1-d)}{d^{2}}
$$

etc. After this step, it is easy to show that the integrands involved in the integral over $r^{\prime}$ are always finite and no additional subtractions are needed. The final result is that upon discretization, the right hand side of the equation is replaced by a set of $N \times N$ matrices labeled by the index $m$ acting on the $N$ 
component vectors $\delta_{m}\left(r_{i}\right)$. We will later discuss in more detail how the discretization works and especially how we treat the boundary conditions both at the tip and at the far tail.

We must perform a similar evaluation of the left hand side of the evolution equation, those terms arising from $\Delta_{\mu}$ given in equation (10). There are two distinct types of terms; there are terms arising from $\Delta_{\mu}$ of the Ivantsov solution which are independent of $\delta_{m}$ and terms linear in $\delta_{m}$. The first set of terms are given by just using the Ivantsov solution $z_{0}(r)$ in equation (12) for the axisymmetric shift. The resulting expression then acts as a source for the linear shape equation.

The terms which depend on $\delta_{m}$ are simple but tedious to compute. The actual details are presented in the Appendix and here we describe only the structure of the final answer. Recall from equation (2) that $\gamma$ has a term explicitly proportional to $\cos 4 \phi$. This means that this side of the equation will couple different $m$ values; if we think of a super matrix with blocks labeled by $m$ and $m^{\prime}$ (each consisting of $N \times N$ matrices), there will be non-zero entries whenever $m^{\prime}=m, m \pm 1$. Each such block can be written schematically as

$$
f_{1}(r) \frac{\partial^{2}}{\partial r^{2}}+f_{2}(r) \frac{\partial}{\partial r}+f_{3}(r)
$$

with the $f$ 's explicitly computed functions. The operators are turned into matrices by finite difference methods, as in the last section.

There are some subtleties which arise near $r=0$. First, we must explicitely set $\delta_{m}(0)=0$ for all $m \neq 0$. This would otherwise give rise to $\Delta_{\mu}$ which diverges quadratically at the tip. We of course cannot set $\partial \delta_{m}(0) / \partial r$ to zero; this is the essence of the solvability mechanism and must be kept free so as to later allow us to fix the unknown parameters governing the solution. To handle this, we modify the derivative piece in expression (19) to be

$$
f_{2}(r)\left(\frac{\partial \delta_{m}(r)}{\partial r}-\frac{\partial \delta_{m}(0)}{\partial r}\right)
$$

exactly as in the axisymmetric case. After this change $\Delta_{*}$ is finite at the tip and the linear problem is well-defined.

In the remainder of this section we return to the study of the axisymmetric problem, this time in the linear approach. Our variables are $\delta_{0}\left(r_{i}\right)$ with the discretization points given as $r_{i}=i r_{\max } / N, i=0$, $N-1$. We derive $N-1$ equations by evaluating both sides of the equation in the manner just described at the points $r_{i}, i=1, N-1$. Note that there is no equation at the tip. The final equation arises from the fixing of the asymptotic form of $\delta_{0}$. In Appendix D we derive the result of Ref. [9] that $\delta_{0} \sim b r$ for large $r$ and explicitly compute $b$. We then choose $\delta_{0}\left(r_{\max }\right)=b r_{\max }$. For further accuracy, we can include the contributions from the integral in the region $r_{\max } \leqslant r^{\prime} \leqslant \infty$ by using this explicit form for the shift.
Table 2. Linearized axisymmetric data, $p=0.1$

\begin{tabular}{llll}
\hline \multicolumn{1}{c}{$\gamma_{0}$} & $N$ & \multicolumn{1}{c}{$r_{\max }$} & \multicolumn{1}{c}{$\delta_{0}^{\prime}(0)$} \\
\hline 0.000185 & 120 & 0.75 & $1.26 \times 10^{-3}$ \\
0.000185 & 180 & 0.75 & $1.18 \times 10^{-3}$ \\
0.000185 & 180 & 1.0 & $1.30 \times 10^{-3}$ \\
0.00019 & 120 & 0.75 & $6.37 \times 10^{-4}$ \\
0.00019 & 180 & 0.75 & $4.88 \times 10^{-4}$ \\
0.00019 & 180 & 1.0 & $6.73 \times 10^{-4}$ \\
0.000195 & 120 & 0.75 & $-3.08 \times 10^{-4}$ \\
0.000195 & 180 & 0.75 & $-1.84 \times 10^{-5}$ \\
0.000195 & 180 & 1.0 & $-5.46 \times 10^{-6}$ \\
\hline
\end{tabular}

The final set of equations are solved by using a standard linear algebra package.

In Table 2, we give the value of $\delta_{0}^{\prime}(0)$ for various values of $\gamma_{0}$, showing the effects of varying the cutoff parameters $r_{\max }$ and $N$. Clearly, the answers being arrived at are fairly insensitive to the exact discretization. We are therefore in a position to extract the selected values of $\gamma$ for different Peclet numbers. Our results are that $\gamma_{0} / p^{2}=0.0194$ at $p=0.1$ and 0.0179 at $p=0.25$, all for $1 \%$ anisotropy. Comparing these numbers to those found in the last section shows that the linear approximation is quantitatively valid at an accuracy level of about $5 \%$. The other anisotropy which we studied, $\epsilon=0.005$, gives rise to agreement at about the same level. The fact that a completely separate computation led to the same answers gives us confidence in the validity of the computer code, the accuracy of our numerical methodology, and the physical nature of the selection mechanism.

\section{A TWO MODE CALCULATION}

After the preliminaries set forth in the last section, we are ready to describe our results for shape selection including the effects of non-axisymmetry. We will present our methodology for the case of general $M$ but the numerical results will be restricted to $M=2$. Inasmuch as the physical anistropy is fourfold, any significant change due to the departure from axisymmetry will be present to this order.

First, we need to discuss the asymptotic form. For the axisymmetric case, we have already seen that there is a term linear in $r$ and a constant which is a priori undetermined. This constant is a trivial consequence of translation invariance in the $\hat{z}$ direction. The asymptotic behavior of the other $m-1$ modes are much less trivial. It turns out that for each and every $m$, there is one undermined parameter. This parameter is the coefficient $f_{m}$ of a term in $\delta_{m}$ which grows as $r^{4 m}$. The reason this coefficient cannot be determined is that the shift $\delta_{m}(r)=f_{m} r^{4 m}$ does not contribute to the integral on the right hand side of the evolution equation. This is proved in Appendix $C$.

Another way of stating the above result is to recognize that the function

$$
\delta(r, \phi)=r^{4 m} \cos 4 m \phi
$$

is an exact zero mode of the Ivantsov zero surface 
tension problem. Now imagine substituting this expression on the left hand side (the $\Delta_{\mu}$ piece) of the shape equation. This substitution produces terms that grow no more rapidly than $r^{4 m-3}$. These terms in turn can be canceled by adding terms of the form $r^{4 m-1}$ to $\delta_{m}$. This process can be continued to all powers of 1/r. (Some of the details of how to do this are presented in Appendix D.) The resulting shift vector $\delta_{m}(r)$ will not necessarily have zero slope at the tip. However, we have already expanded our space of functions to allow this to occur. We therefore have found an exact zero mode of our full linear problem, including the surface energy term. This conclusion can be verified directly by studing the spectral problem of the linearization operator and showing that there is a mode at zero eigenvalue whose eigenvector has the expected behavior.

So, we have discovered $M-1$ additional degrees of freedom in the problem as stated. We also have discovered that our matrix is singular and must be handled with care. We will return to this momentarily. First, though, we should note that after finding $\delta_{m}(r)$, the next step in the calculation will be to impose the solvability conditions at the tip. But there are exactly $M$ different conditions since we require $\delta_{m}^{\prime}(0)=0$ for all $m$ ! These serve to fix the $M$ unknowns and in the process to determine the allowed shape and growth velocity. The extra degrees of freedom which appear upon allowing nonaxisymmetric shapes are exactly what is necessary to allow us to find a solution which is completely smooth near the tip.

We therefore proceed to study the case $M=2$ as follows. We parameterize the shape by explicitly adding a zero mode piece $\delta_{1}^{(0)}$ to the as yet unknown shift. We write for the fourfold piece

$$
\delta_{1}\left(r_{i}\right)=f_{1} \delta_{i}^{(0)}\left(r_{i}\right)+\left(1+\frac{r_{i}^{3}}{p^{3}}\right) \delta_{1, i}
$$

for the discrete points $r_{i}=i r_{\max } / N$, for $i=0, N-1$. At all $r$ past $r_{\text {max }}, \delta_{1, i}$ is taken to equal zero. For the axisymmetric part we set

$$
\delta_{0}\left(r_{i}\right)=\left(1+\frac{r_{i}^{2}}{p^{2}}\right) \delta_{0, i}
$$

We can use the fact that the asymptotic growth rate is linear in $r$ to demand that all $\delta_{0, i}$ for $i \geqslant N$ are zero. We demand $\delta_{m}(0)=0$, reducing the number of unknowns to $N-1$ for both $m$ values. Finally, we must take into account the singular nature of the matrix by imposing the additional constraint that the actual $r^{4}$ growth coefficient is exactly $f_{1}$; in practice this amounts to adding one additional boundary condition at the last point, fixing not only the function but also its derivative. This constraint equation means we need one more variable, which in standard fashion takes the form of a lagrange multiplier. This lagrange multiplier ensures that the source term is indeed orthogonal to the zero mode subspace (the Fredholm alternative condition). Once this is done, the un- knowns are determined by solving the $N-1$ equations which arise upon evaluating both sides of the linearized steady-state equation at the points $r_{i}, i=1$. $N-1$. Just as before, there are no equations at the tip.

The above calculation then determines the shift vector $\delta(r, \phi)$ as a functional of the two unknowns $\gamma_{0}$ and $f_{1}$. This then determines the slopes at the tip of the axisymmetric and fourfold components of the shape. The accuracy of the answers is at least as good as that quoted previously for the axisymmetric linearized problem (see Table 2); in principle, any desired accuracy could easily be obtained by increasing $N$ and $r_{\max }$. A consistency check is provided by the value of the lagrange multiplier. In particular, the linearization we have performed is only valid if the source (the Gibbs-Thomson temperature change for the Ivantsov parabola) is orthogonal to the (adjoint) zero mode. If this is the case, the lagrange multiplier will be zero in the limit of infinite resolution, infinite box size and no computer roundoffs. Our numerical results do indeed indicate this to be true. We do not, however, have an analytic proof of this orthogonality.

In Fig. 2 we have plotted $\delta_{0}^{\prime}(0)$ vs $\gamma_{0}$ at different values of the parameters $f_{1}$, at $p=0.25$. We note that the root is almost completely insensitive to the value of $f_{1}$. In fact, the root occurs at almost precisely the same point $(0.0011)$ as it did in the previous two sections. In Fig. 3 we set $\gamma_{0}$ at this selected value and show $\delta_{1}^{\prime}(0)$ as a function of $f_{l}$. There is a root at a small but non-zero value. The set $\gamma_{0}, f_{1}$ determine the selected shape for steady-state motion.

One of the more surprising features of our solution is that the shift vector grows at a faster rate (as a function of $r$ ) than does the Ivantsov paraboloid. Since the coefficient is small, the needle crystal will nonetheless remain approximately axisymmetric for quite a long distance away from the tip. In Fig. 4 we present the final shape of the solution, seen from two different viewing angles. One might be able to make a quantitative comparison between this picture and the experimentally observed succinonitrile dendrite. but this has not been attempted.

In Table 3, we present data for the selected parameters for different Peclet numbers, at all $\epsilon=0.01$. Of

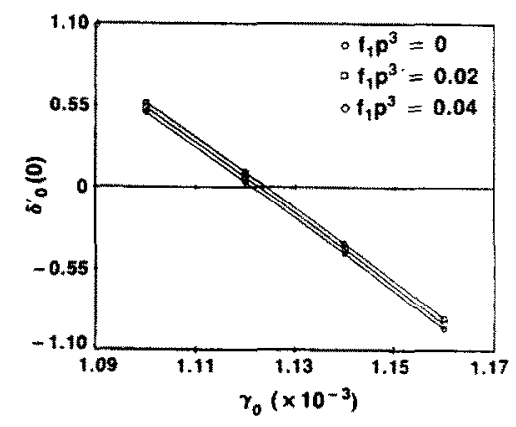

Fig. 2. $\delta_{0}^{\prime}$ at tip vs $\gamma_{0}$ at several values of fourfold parameter $f_{1} p^{3}$, all at $p=0.25$. 


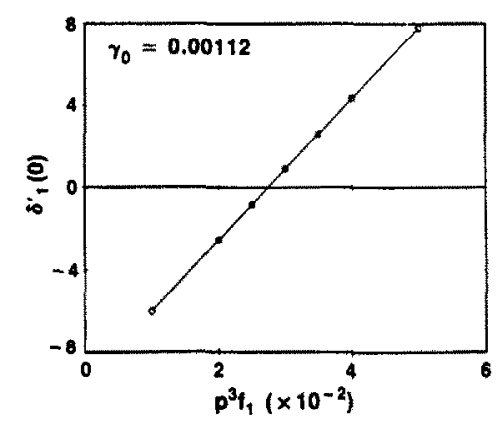

Fig. 3. $\delta_{1}$ at tip vs $f_{1} p^{3}$ at the selected value of $\gamma_{0}$ at $p=0.25$.

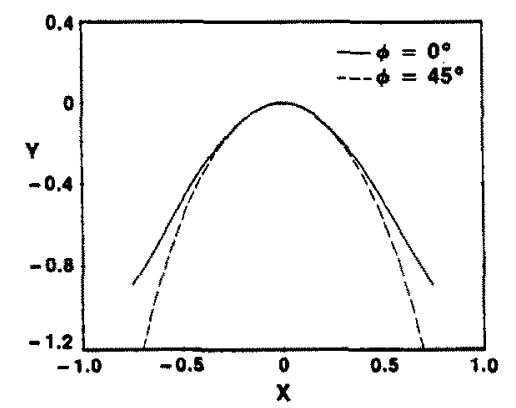

Fig. 4. Final shape of the $p=0.25$ needle crystal, viewed in the planes $\phi=0$ and $\phi=\pi / 4$.

Table 3. Non-axisymmetric selected $\gamma_{0}$,

\begin{tabular}{cll}
\multicolumn{3}{c}{$f_{1}$} \\
\hline$p$ & \multicolumn{1}{c}{$\gamma_{0}^{*}$} & \multicolumn{1}{c}{$f_{1} p^{3}$} \\
\hline 0.05 & 0.0000497 & 0.0275 \\
0.1 & 0.000193 & 0.0257 \\
0.25 & 0.00112 & 0.0250 \\
\hline
\end{tabular}

particular interest is the approximate scaling $\gamma_{0} \sim \sigma^{*} p^{2}$, with $\sigma^{*}=0.02$. This is the same number as we found in the axisymmetric version, demonstrating that this result does indeed provide a valid estimate for the full three-dimensional system. A possible explanation of this indifference to non-axisymmetric terms is that the $\cos 4 \phi$ piece of the surface energy $\gamma$ is proportional to $\sin ^{4} \theta$ and hence is relatively unimportant near the tip, which is where the selection occurs.

So, our final chain of reasoning goes as follows. We can linearize the steady-state equation around the Ivantsov solution without any significant loss in accuracy; this was seen most explicitly in a comparison of linear and non-linear approaches to the axisymmetric limit. Next, we truncate the full linear problem to just two modes, arguing that any important effects due to the full three-dimensional nature of the shape will show up most strongly in a $\cos 4 \phi$ term. Finally, we use the mode truncated equation to demonstrate that the result of the axisymmetric calculation is in fact quite accurate, perhaps as good as $1-2 \%$. In the end, all three estimates of $\sigma^{*}$ are mutually consistent. And, the answer we arrive at agrees with the experimental determination by Huang and Glicksman [13] $\left(\sigma^{*}=0.019\right)$ to within a factor of two or so, and possibly much better depending on the exact value of the anisotropy.

\section{SIDEBRANCH WAVELENGTH}

The previous sections of this work have shown how to compute the velocity and tip shape of the selected dendritic crystal. Physical dendrities consist, of course, of sidebranches in addition to the steady-state structure. It is as yet unclear even in two dimensions whether sidebranching is due to a subcritical bifurcation away from the steady-state or is generated by external noise. There is some evidence [26] that sidebranches are inherently noisy, with the concept of a unique wavelength true only as a first approximation. In any event, there is a peak in the spectrum of sidebranch oscillations and in any physical realization this peak will almost certainly have non-zero width.

In a previous paper [14], we showed how one could arrive at an estimate for this peak sidebranching wavelength. The idea is to consider the effect of adding noise to the tip region of the steady-state structure. This noise will be amplified selectively and there will be a maximally amplified frequency which will dominate the sidebranching whenever the noise is sufficiently broadband in nature. This calculation predicts wavelengths that are in agreement with the numerical simulations of two dimensional dendritic growth [8].

We now perform a similar estimate for the three dimensional case of interest here. For simplicity, we will restrict ourselves to the axisymmetric case; just as in earlier sections, we expect that the inclusion of nonsymmetric terms will not significantly alter the estimate. To do this, we start from the time dependent evolution equation, equation (3) and now let

$$
\begin{aligned}
z(r, \phi, t) & =-\frac{r^{2}}{2 p}+\delta(r, t) \\
v_{n} & =(\hat{n} \cdot \hat{z})(v+\delta) .
\end{aligned}
$$

We use the quasistatic approximation which allows us to ignore the time dependence of $\delta$ only in its contribution to the velocity. Formally, this amounts to solving the heat diffusion equation by first shifting to the moving frame of the steady-state solution and then dropping the remaining time derivative. This approach can be shown to be valid for small Peclet number. This approximation leads to the stability equation

$$
\begin{aligned}
-\Delta_{\mu \prime}^{(1)}[\delta] & \\
= & \int_{0}^{2 \pi} \frac{\mathrm{d} \phi}{2 \pi} \int_{0}^{\infty} r^{\prime} \mathrm{d} r^{\prime} \frac{\mathrm{e}^{z_{0}\left(r^{\prime}, \phi\right)-z_{0}(r, \phi)-d}}{d} \\
& \times\left\{\left[\delta\left(r^{\prime}\right)-\delta(r)\right]\right. \\
& \left.\times\left[1-\frac{z_{0}\left(r^{\prime}\right)-z_{0}(r)}{d}\left(1+\frac{1}{d}\right)\right]+i \omega \delta\left(r^{\prime}\right)\right\}
\end{aligned}
$$

where we have assumed a single frequency time 


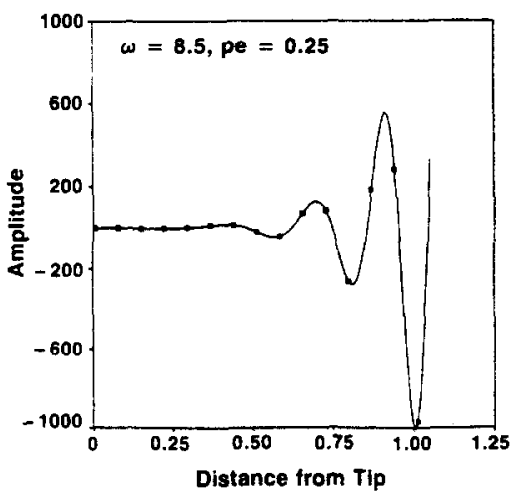

Fig. 5. Perturbation shape for imposed noise at the tip, $p=0.25, \omega=8.5$.

dependence for the perturbation and $\omega$ is measured in units of $v^{2} / 4 D$. By $\Delta_{\mu}^{(1)}$ we mean that portion of the $\Delta_{\mu}$ which depends linearly on $\delta$, as discussed in Section 4.

We solve this equation in a very similar manner to how we did in Section 4. We discretize the curve and write a matrix representation for the equation by evaluating both sides at the discrete set of observation points. The variables are $\delta_{i} \equiv \delta\left(r_{i}\right), i=0$, $N-1$. We add noise to the tip by fixing $\delta(0)=1$, while maintaining $\delta^{\prime}(0)=0$ via proper choice of $\delta_{1}$ and $\delta_{2}$. We can then plot the function $\delta(r)$ as a function of diatance away from the tip for some particular frequency.

A typical curve resulting from this calculation is shown in Fig. 5. The perturbation oscillates with increasing amplitude and approximately obeys $\omega=k$ for spatial wavevector $k$. This type of wave is stationary in the laboratory frame of reference and has wavelength $\lambda=2 \pi / \omega$.

The basic method of selecting the sidebranch wavevector relies upon the fact that different frequencies will be amplified at different rates. In particular, there is a maximally amplified frequency $\omega^{*}$ which, for all else being equal, will be the dominant sidebranching mode. In Fig. 6 we show graphs of the amplification

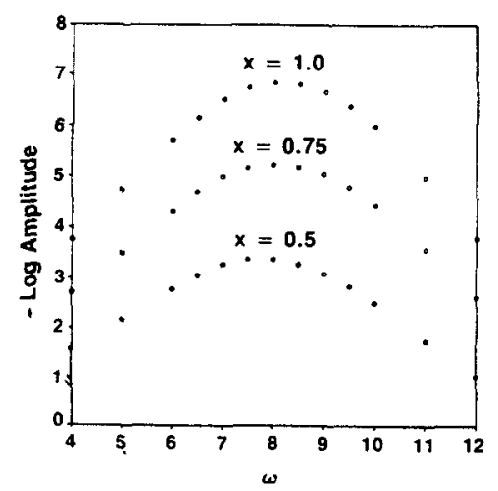

Fig. 6. Log amplification vs frequency, at several distances from the tip, at $p=0.25$. versus frequency at several positions away from the tip; note that the fact that the peak does not shift very much means that $\omega^{*}$ is well-defined. Once this is done, the wavelength is given by $\lambda^{*}=2 \pi / \omega^{*}$. In the units we are using, the diffusion length is 1 and the tip radius is the Peclet number $p$. Therefore our final prediction for the ratio of sidebranch wavelength to tip radius is given by

$$
\frac{\lambda^{*}}{R_{\text {tip }}}=\frac{2 \pi}{p \omega^{*}} .
$$

We determined $\omega^{*}$ in this manner for Peclet numbers of $0.05,0.1$ and 0.25 , all at $\epsilon=0.01$; we obtained $41.0,21.0$ and 8.5 respectively. The resultant values for this ratio are all about 2.9 , to an accuracy of $10 \%$. Note that this calculation predicts that the sidebranch wavelength scales as the tip radius as a function of undercooling. Both the scaling and the ratio agree with the measurements in succinonitrile [13]!

We obviously could do more to improve this calculation. It would be nice to include the effects of non-axisymmetric terms as well as the effects of going beyond the linear order and coupling the stability shift to the steady-state shift computed earlier. Nevertheless, it is reassuring that this simple approach succeeds in capturing fairly accurately the typical length scale present in the sidebranch train. A further challenge would be to try to predict in more detail the entire temporal structure of the branches to compare to the experiments of Ref. [26]. This would be easier if there were more information about the equilibrium crystal shape of ammonium chloride, which is the material used in those studies.

\section{CONCLUSIONS}

Over the past several years, a new approach to interfacial pattern formation has succeeded in explaining many of the qualitative features of these structures. The only quantitative test to date [27], though, was in the Saffman-Taylor finger [28], a steady-state structure formed during multiphase fluid displacement. No quantitative comparison has been possible for solidification, either because of the three dimensional nature of the growth process or because of the lack of knowledge about material parameters or, most often, both.

In this work, we have taken the first step towards applying our new "microscopic solvability" approach to the case of actual crystal growth. We studied free space dendrites in cubically anisotropic substances with a specific focus on succinonitrile. For this material, Glicksman and coworkers have, over the years, measured all the relevant material constants, carefully studied dendritic growth as a function of undercooling, and shown that kinetic effects not present in the Gibbs-Thomson equilibrium condition are negligible. Patterns in succinonitrile therefore serve as a vital test for our theory with no adjustable parameters or unknown constants to mask any inadequacy. 
We have computed $\sigma^{*}$ as well as $\lambda_{\text {sB }} / R_{\text {tip }}$ for the case of $1 \%$ anisotropy, obtaining results that agree with the corresponding measured values. Although this was the anisotropy value quoted in the initial studies on succinonitrile, later work suggests that the actual value is closer to half that. Our theory is crucially dependent on anisotropy to generate steadystate solutions and therefore our results are extremely sensitive to changes in the measured value of $\epsilon$. What we can say with certainty at present is that the growth velocity and sidebranch spacing are in qualitative agreement with the experiments and can be made to agree quantitatively by using the $1 \%$ value. Another possibility is that we need to include higher order anisotropies which have magnified effects in Gibbs-Thomson condition, as compared with the deviation of equilibrium shape from a sphere; this is due to the extra derivatives appearing in equation (12). This will certainly be true of materials that are more anisotropic than succinonitrile.

What about other materials? There have been many generations of dendritic crystal growth experiments, but only in a few cases were they sufficiently controlled to permit direct comparision. A still puzzling case is the experiment on pivalic acid [22]; this substance seems to be about 10 times more anisotropic than succinonitrile but $\sigma^{*}$ is only slightly larger. This could be due to kinetic effects not currently contained in the theoretical analysis, but the scaling with Peclet number at small undercooling seems to indicate that these effect are small.

One possible explanation for the results on pivalic acid is, as we have already mentioned, that the anisotropy cannot be described simply by a cubic term. Some natural choices are the six fold term

$$
\gamma_{6} \sim \frac{x^{2} y^{2} z^{2}}{r^{6}} \sim(1-\cos 4 \theta)(1-\cos 4 \phi)
$$

or eightfold terms proportional to $\cos 8 \phi$. Note that the possible presence of terms like the abovementioned sixfold piece requires measurements in more than one plane; $\gamma_{6}$ vanishes at $\theta=\pi / 2$ and would not show up in the pictures taken in the principal plane.

Previous theories of dendrite growth did not care in an essential way about the presence of crystallinity. In fact, the standard predictions of $\sigma^{*}$ by stability criteria were done in completely isotropic systems. This weakness of the theory became evident when simulations [29] and experiments [30] clearly demonstrated that crystal anisotropy is absolutely essential for obtaining dendrites. Nevertheless, the influence of the theory has meant that very little attention has been paid to detailed maps of the equilibrium surface energy versus angle. This will have to remedied before we can proceed with more detailed tests of the current approach.

Aside from the case of solidification from the pure melt, there are other systems which could be studied with the methodology developed here. It would only require minor modifications to include the effects of unequal diffusion constants in the two phases or the effects of diffusing chemical impurities. A more difficult task awaits those who attempt to study directional solification [31]. Although there are some indications that a solvability mechanism is present [32], there are still many unresolved issues and we are far from quantitative predictions. The same should be said regarding lamellar eutectics [33] where even less has been accomplished to date.

It is worthwhile to end with a brief historical aside. The problem of free space dendritic growth has been seriously studied for many decades. The efforts improved over time from the hopelessly inadequate maximal velocity prediction to the $a d$-hoc but qualitatively successful marginal stability hypothesis, culminating in the present theory of shape selection via solvability. The major breakthrough which led to the formulation of this approach was made through the study of simplified local models of dendritic growth [34]. It turned out that these models did somehow accurately refiect the subtleties of pattern selection in a vastly simpler setting. Although these models have in a certain sense outlived their usefulness, in a broader view they remain as a paradigm of the usefulness of "toy" models as a means of generating new ideas which can then lead to quantitative predictions for realistic physical systems.

Acknowledgements - One of us (H.L.) would like to acknowledge useful conversations with $\mathrm{M}$. Glicksman regarding his anisotropy measurements, J. Langer regarding the functional form of the Gibbs-Thomson effect, and J. Collins about aspects of the non-axisymmetric calculation.

\section{REFERENCES}

1. For a recent review, see D. Kessler, J. Koplik and $\mathbf{H}$. Levine, Adv. Phys. To be published.

2. D. Kessler and H. Levine, Phys. Rev. B33, 7687 (1986). 3. D. Meiron, Phys. Rev. A33, 2704 (1986).

4. D. Kessler, J. Koplik and H. Levine, "Pattern Formation Far From Equilibrium: The Free Space Dendritic Crystal", "Pattern Defects and Microstructures", (edited by D. Walgraet), NATO ASI Series E, Vol. 121. Nijhoff (1987).

5. D. Hong and J. S. Langer, unpublished.

6. B. Caroli, C. Caroli, B. Roulet and C. Misbah, $J$. Physique 48, 547 (1981).

7. M. Ben-Amar and Y. Pomeau, Europhys. Lett. 2, 307 (1986).

8. Y. Saito, G. Goldbeck-Wood and H. MullerKrumbhaar, Phys. Rev. Lett. 58, 1541 (1987).

9. D. Kessler, J. Koplik and H. Levine, Phys. Rev. A33, 3352 (1986).

10. A. Barbieri. D. Hong and J. Langer, Phys. Rev. A35, 1541 (1987)

11. M. Ben-Amar and Y. Pomeau, Physiochemical Hydrodynamics. To be published.

12. M. Ben-Amar and B. Moussallam, Physica 25D, 155 (1987).

13. S. C. Huang and M. E. Glicksman, Acta metall. 79, 701, 717 (1981); M. E. Glicksman, Mater. Sci. Engng 65, 45 (1984).

14. D. Kessler and H. Levine, Europhys. Lett. 4, 215 (1987). For a discussion of possible causes of sidebranching, see 
O. Martin and N. Goldenfield, Phys. Rev. A35, 1382 (1987).

15. D. P. Woodruff. The Solid-Liquid interface Cambridge Univ. Press (1973); J. S. Langer, rev. Mod. Phys. 52, 1 (1980).

16. P. Pelce and Y. Pomeau, Stud. appl. Math. 74, 245 (1986)

17. G. P. Ivantsov, Dokl. Akad. Nauk SSSR 58, 567 (1947).

18. W. W. Mullins, in Metal Surfaces. Am. Soc. Metals, Metals Park, Ohio (1963).

19. J. M. Vanden-Broeck, Phvs. Fluids 26, 2033 (1983).

20. P. W. Voorhees, S. R. Coriell, G. B. McFadden and R. F. Sekerka, J. Cryst. Growth 67, 425 (1984), and references therein

21. M. E. Glicksman, R. J. Schaefer and J. D. Ayers, Metall. Trans. A7, 1747 (1976); S. C. Huang and M. E. Glicksman, Acta metall. 79, 701, 717 (1981); M. E. Glicksman, Mater. Sci. Engng. 65, 45 (1984); in these papers, the crystal anisotropy of succinonitrile is quoted to be about 1\% (see e.g. Fig. 4 on p. 722 of Huang and Glicksman).

22. M. E. Glicksman and N. B. Singh, in Special Technical Publication 890, ASTM Philadelphia, Pa (1986).

23. Digital imaging has recently been used to obtain an accurate measurement of the anisotropy for ammonium chloride (A. Dougherty, private communication).

24. D. Kessler and H. Levine, Phys. Rev. Lett. 57, 3069 (1986).

25. J. S. Langer and H. Müller-Krumbhaar, Acta metall. 26, 1681, 1689, 1697 (1978).

26. A. Dougherty, P. Kaplan and J. Gollub, Phys. Rev. Lett. 58, 1652 (1987).

27. P. Tabeling, G. Zocchi and A. Libchaber, J. Fluid Mech. to be published; S. Sarkar and D. Jasnow, Pittsburgh preprint (1987).

28. P. G. Saffman and G. J. Taylor, Proc. R. Soc. A245, 312 (1952).

29. D. Kessler, J. Koplik and H. Levine, Phys. Rev. A30, $2820(1984)$

30. See for example, G. Radnoczi, R. Viscek, L. Sander and D. Grier, Univ. of Michigan preprint (1986).

31. K. Somboonsuk, J. T. Mason and R. Trivedi, Metall. Trans. 15A, 967 (1984).

32. A. Karma, Phys. Rev. Lett. 57, 858 (1986); for a dissenting view, see T. Dombre and V. Hakim, Ecole Normale preprint (1987).

33. W. Kurz and D. J. Fisher, Fundementals of Solidification. Trans Tech Publications, Aedernannsdorf, Switzerland (1984).

34. For a review, see D. A. Kessler, J. Koplik and H. Levine, Physiochem. Hydro. 6, 507 (1985).

35. I. S. Gradshteyn and I. M. Ryzhik. Tables of Integrals, Series and Products. Academic Press. New York (1980).

36. W. Van Saarloos. J. Weeks and G. Kotliar, Bell Labs preprint (1986).

\section{APPENDIX A}

We wish to derive the relationship between the equilibrium crystal shape and the surface energy, for small anisotropy. Let us assume that

$$
\gamma(\theta, \phi)=\gamma_{0}[1+\epsilon g(\theta, \phi)]
$$

for small $\epsilon$. We parameterize the equilibrium crystal shape in spherical coordinates

$$
\rho(\theta, \phi)=\rho_{0}[1+\epsilon h(\theta, \phi)] .
$$

To proceed, we need to evaluate the expression for $\Delta_{\mu}$, equation (10), to first order in $\epsilon$ and then set $\Delta_{\mu}=0$. The terms which depend on $g$ to this order are

$$
\begin{aligned}
-\epsilon \gamma_{0}\left[\frac{-2 g}{\rho_{0}}+\frac{\partial^{2} g}{\partial \theta^{2}} \frac{z_{0}^{\prime \prime}}{\left(1+z_{0}^{\prime 2}\right)^{3 / 2}}\right. & +\frac{\partial^{2} g}{\partial \phi^{2}} \frac{\left(1+z_{0}^{\prime 2}\right)^{1 / 2}}{r z_{0}^{\prime}} \\
& \left.-\frac{\partial g}{\partial \theta} \frac{1}{r\left(1+z_{0}^{\prime} 2\right)^{1 \cdot 2}}\right] .
\end{aligned}
$$

Using the relationships

$$
z_{0}(r)=\sqrt{\rho_{0}^{2}-r^{2}},
$$

and $\sin \theta=r / \rho_{0}$, we can rewrite (A2) as

$$
-\epsilon \gamma_{0}\left[\frac{-2 g}{\rho_{0}}-\frac{\partial^{2} g}{\partial r^{2}} \frac{\rho_{0}^{2}-r^{2}}{\rho_{0}}-\frac{\partial^{2} g}{\partial \phi^{2}} \frac{\rho_{0}}{r^{2}}-\frac{\partial g}{\partial r} \frac{\rho_{0}^{2}-2 r^{2}}{\rho_{0} r}\right] \text {. }
$$

Next, we evaluate the $h$ dependent pieces. To do this, we must take into account the parameterization shift in the $(z, r, \phi)$ system caused by the normal shift $h$. In particular, the shift not only changes $z$ but also redefines the coordinates. It is easiest to change temporarily to cartesian coordinates; we can write explicitly the shifted variables

$$
\begin{aligned}
& \bar{z}=z+\frac{1}{\sqrt{1+z_{0}^{\prime 2}}} \rho_{0} h \\
& \tilde{x}=x-\frac{1}{\sqrt{1+z_{0}^{\prime 2}}} \frac{\partial z_{0}}{\partial x} \rho_{0} h \\
& \tilde{y}=y-\frac{1}{\sqrt{1+z_{0}^{\prime 2}}} \frac{\partial z_{0}}{\partial y} \rho_{0} h .
\end{aligned}
$$

Consider the change in

$$
\frac{\partial z}{\partial x}
$$

From the above we can calculate that

$$
\begin{aligned}
\frac{\partial \dot{z}}{\partial \dot{x}}= & \frac{\partial z}{\partial x}+\frac{\partial}{\partial x}\left(\frac{\rho_{0} h}{\sqrt{1+z_{0}^{\prime 2}}}\right) \\
& +\frac{\partial z_{0}}{\partial x} \frac{\partial}{\partial x}\left(\frac{\rho_{0} h}{\sqrt{1+z_{0}^{\prime 2}}} \frac{\partial z_{0}}{\partial x}\right) \\
& +\frac{\partial z_{0}}{\partial y} \frac{\partial}{\partial x}\left(\frac{\rho_{0} h}{\sqrt{1+z_{0}^{\prime 2}}} \frac{\partial z_{0}}{\partial y}\right) .
\end{aligned}
$$

A similar computation for the $y$ derivative and evaluation of the derivatives leads to the result

$$
\tilde{\vec{\nabla}} z=\vec{\nabla} z+\sqrt{1+\left(\bar{\nabla} z_{0}\right)^{2}} \rho_{0} \vec{\nabla} h .
$$

Substituting this expression into the first term in equation (10) for the Gibbs-Thomson shift allows us to identify the first set of terms linear in $h$

$$
\epsilon \gamma_{0}\left[-\frac{\partial^{2} h}{\partial r^{2}} \frac{\rho_{0}^{2}-r^{2}}{\rho_{0}}+\frac{\partial^{2} h}{\partial \phi^{2}} \frac{\rho_{0}}{r^{2}}+\frac{\partial h}{\partial r} \frac{\rho_{0}^{2}-3 r^{2}}{\rho_{0} r}\right]
$$

We also must compute the change due to the altered $\vec{\nabla}$ appearing in the same term. This piece is easily seen to be the correction to

$$
1 / \rho_{0}\left(\frac{\partial x}{\partial \tilde{x}}+\frac{\partial y}{\partial \tilde{y}}\right)
$$

Evaluating this term, keeping the linear term in $h$ and adding to (A6) leads to the final expression

$$
\epsilon \gamma_{0}\left[\frac{-2 h}{\rho_{0}}-\frac{\partial^{2} h}{\partial r^{2}} \frac{\rho_{0}^{2}-r^{2}}{\rho_{0}}-\frac{\partial^{2} h}{\partial \phi^{2}} \frac{\rho_{0}}{r^{2}}-\frac{\partial h}{\partial r} \frac{\rho_{0}^{2}-2 r^{2}}{\rho_{0} r}\right] .
$$

Comparing this to (A3), we must clearly have $h=g$ as the equilibrium condition.

This result allows us to estimate $\epsilon$ for cubic crystals by measuring $r(\theta)$ in one plane. For such a crystal, $\gamma$ is described by equation (2) and therefore by the results of this appendix

$$
\begin{array}{r}
r(\theta, \phi=0)=r_{0}\left[1+4 \epsilon\left(\cos ^{4} \theta+\sin ^{4} \theta\right)\right] \\
=r_{0}[1+3 \epsilon+\epsilon \cos (4 \theta)]
\end{array}
$$


we can drop the small correction (the $3 \epsilon$ ) in the isotropic piece. Then the measured four-fold anisotropy in the $r(\theta)$ plot directly determines the anisotropy. Glicksman and coworkers $[21,22]$ have shown that for succinonitrile, $r=r_{0}(1+\epsilon \cos 4 \theta)$, with $0.005<=\epsilon<=0.01$.

\section{APPENDIX B}

In this Appendix we present more details of the linearization described in Section 4 of the text. We start with the right hand side of the evolution equation. We must perform the integrals

with

$$
I_{m, n} \equiv \int_{0}^{2 \pi} \frac{\mathrm{d} \phi^{\prime}}{2 \pi b^{n}} \frac{\cos 4 m \phi^{\prime}}{\left[\sqrt{\left.1-a \sin ^{2}\left(\frac{\phi-\phi^{\prime}}{2}\right)\right]^{n}}\right.}
$$

$$
a=\frac{4 r r^{\prime}}{b^{2}}, \quad b=\sqrt{\left(r+r^{\prime}\right)^{2}+\left[z_{0}(r)-z_{0}\left(r^{\prime}\right)\right]^{2}}
$$

for the cases $n=1,3$. Once these are done, the right hand side of the shape equation picks up terms of the form which follows from equation (15)

$$
\begin{aligned}
\left\{I_{1, m}-\right. & {\left.\left[z_{0}(r)-z_{0}\left(r^{\prime}\right)\right]\left(I_{3, m}-1 / 2 I_{1, m}\right)\right\} \delta_{m}\left(r^{\prime}\right) } \\
& -\left\{I_{1,0}-\left[z_{0}(r)-z_{0}\left(r^{\prime}\right)\right]\left(I_{3,0}-1 / 2 I_{1,0}\right)\right\} \delta_{m}(r)
\end{aligned}
$$

in addition to the piece from the remaining finite integrals (given later). Note that the $n=2$ term has cancelled out of this expression and need not be computed.

These integrals are all elliptic functions multiplied by polynomials. Let us shift $\phi^{\prime}$ by $\phi$ and also absorb a factor of $1 / 2$. This leads to

$$
I_{m, n}=\cos 4 m \phi \int_{0}^{\pi / 2} \frac{2 d \phi^{\prime}}{b^{n} \pi} \frac{\cos 8 m \phi^{\prime}}{\left(\sqrt{1-a \sin ^{2} \phi^{\prime}}\right)^{n}} .
$$

It is always possible to express $\cos 8 m \phi^{\prime}$ as a power series of the form

$$
\cos 8 m \phi^{\prime}=\sum_{k=0}^{4 m} c_{k} \cos ^{2 k} \phi^{\prime}
$$

and each integral can be handled separately. For $n=1$, we can use the recursion formula (see Gradshteyn and Ryzhik [35])

$$
\begin{aligned}
\int_{0}^{\pi / 2} \mathrm{~d} \phi^{\prime} & \frac{\cos ^{2 k} \phi^{\prime}}{\sqrt{1-a \sin ^{2} \phi^{\prime}}}=\left(\frac{2 k-3}{2 k-1}\right)\left(\frac{1-a}{a}\right) \\
& \times \int_{0}^{\pi / 2} \mathrm{~d} \phi^{\prime} \frac{\cos ^{2 k-1} \phi^{\prime}}{\sqrt{1-a \sin ^{2} \phi^{\prime}}}+\left(\frac{2 k-2}{2 k-1}\right)\left(\frac{2 a-1}{a}\right) \\
& \times \int_{0}^{\pi / 2} \mathrm{~d} \phi^{\prime} \frac{\cos ^{2 k-2} \phi^{\prime}}{\sqrt{1-a \sin ^{2} \phi^{\prime}}}
\end{aligned}
$$

For $k=0$, the integral is the complete elliptic function $K(\sqrt{a})$ and for $k=2$, the difference of elliptic functions

$$
\frac{1}{a} E(\sqrt{a})-\frac{1-a}{a} K(\sqrt{a}) \text {. }
$$

For small values of $a$, it is useful to recast these formula in terms of hypergeometric functions; one can prove that $b l_{m, 1}$ equals

$$
\begin{aligned}
{\left[\frac{\Gamma(4 m+1 / 2)}{\Gamma(1 / 2)}\right]^{2} } & \frac{a^{4 m}}{\Gamma(8 m+1)} \\
& \times F(4 m+1 / 2,4 m+1 / 2 ; 8 m+1 ; a)
\end{aligned}
$$

A similar set of formulas is valid for $n=3$. The recursion formula (B4) is replaced by

$$
\begin{aligned}
& \int_{0}^{\pi / 2} \mathrm{~d} \phi^{\prime} \frac{\cos ^{2 k} \phi^{\prime}}{\left(\sqrt{\left.1-a \sin ^{2} \phi^{\prime}\right)^{3}}\right.}=\left(\frac{1-a}{a}\right) \\
& \quad \times \int_{0}^{\pi / 2} \mathrm{~d} \phi^{\prime} \frac{\cos ^{2 k-4} \phi^{\prime}}{\left(\sqrt{\left.1-a \sin ^{2} \phi^{\prime}\right)^{3}}\right.}+\left(2-\frac{2 k-2}{(2 k-3) a}\right) \\
& \quad \times \int_{0}^{\pi / 2} \mathrm{~d} \phi^{\prime} \frac{\cos ^{2 k-2} \phi^{\prime}}{\left(\sqrt{\left.1-a \sin ^{2} \phi^{\prime}\right)^{3}}\right.} .
\end{aligned}
$$

This is the supplemented by the results for $k=0$. $E(\sqrt{a}) /(1-a)$ and for $k=1,1 / a[K(\sqrt{a})-E(\sqrt{a})]$. There is also an expression in terms of hypergeometric functions

$$
\begin{aligned}
& \frac{\Gamma(4 m+1 / 2) \Gamma(4 m+3 / 2)}{\Gamma(1 / 2) \Gamma(3 / 2)} \frac{a^{4 m}}{\Gamma(8 m+1)(1-a)} \\
& \times F(4 m-1 / 2,4 m+1 / 2 ; 8 m+1 ; a)
\end{aligned}
$$

This completes the evaluation of the required integrals.

It is important to understand the singularities in (B2) near $r \sim r^{\prime}$. This region of integration corresponds to $a \sim 1+O\left[\left(r-r^{\prime}\right)^{2}\right]$. The $I_{3, m}$ are all quadratically singular, being proportional to $1 /(1-a)$. This singularity is multiplied by a prefactor

$$
\left[\delta_{m}\left(r^{\prime}\right)-\delta_{m}(r)\right]\left[z_{0}(r)-z_{0}\left(r^{\prime}\right)\right]
$$

which vanishes quadratically. The limiting process gives rise to the value of the integral equal to

$$
\frac{\frac{\partial z_{0}(r)}{\partial r} \frac{\partial \delta_{m}(r)}{\partial r}}{1+\left(\frac{\partial z_{0}(r)}{\partial r}\right)^{2}}
$$

There is also a finite piece arising from $\delta_{m}(r)\left(I_{1, m}-I_{1,0}\right)$. There is therefore no need to perform additional subtractions for the remaining integral over $r^{\prime}$.

The parts we have just evaluated must be added to the contribution of the residual terms remaining after the subtractions. If we define

$$
\begin{aligned}
R_{m . n} \equiv \int_{0}^{2 \pi} \frac{\mathrm{d} \phi^{\prime}}{2 \pi b^{n}} \frac{\cos 4 m \phi^{\prime}}{d^{n}} & \times\left[\mathrm{e}^{-d}-\mathrm{e}^{-d b^{j}} \sum_{j \neq 0}^{m-1}(-d)^{j} / j !\right]
\end{aligned}
$$

with

$$
d=\sqrt{b-a b \sin ^{2}\left(\frac{\phi-\phi}{2}\right)}
$$

and $d_{0}=d\left(\phi=\phi^{\prime}+\pi / 2\right)$, the residual contribution is

$$
\begin{aligned}
\left\{R_{1, m}-\right. & {\left.\left[z_{0}(r)-z_{0}\left(r^{\prime}\right)\right]\left(R_{3, m}+R_{2, m}\right)\right\} \delta_{m}\left(r^{\prime}\right) } \\
& -\left\{R_{1,0}-\left[z_{0}(r)-z_{0}\left(r^{\prime}\right)\right]\left(R_{3,0}+R_{2,0}\right)\right\} \delta_{m}(r) .
\end{aligned}
$$

All these pieces are explicitly finite and the integral over $\phi^{\prime}$ is done via the trapezoidal rule. The convergence in the number of points is exponential since we are dealing with a periodic function, and in practice a small number of points $(\sim 20)$ provides extremely accurate results.

We now turn to the left hand side of the equation which involves $\Delta \mu$. In principle, this is quite straightforward; all we need do is substitute the above expression for $z$ into the general formula for $\Delta_{z}$ given in equation (10) and expand everything to linear order in $\delta_{m}$. This involves the evaluation of the changes in various constructs

$$
\begin{aligned}
\vec{\nabla} z= & \hat{r}\left(-r / p+\sum \delta_{m}^{\prime} \cos 4 m \phi\right) \\
& +\hat{\phi} \sum-4 m / r \delta_{m} \sin 4 m \phi \\
\tilde{\theta}= & \tan ^{-1}(r / p)-\frac{1}{1+r^{2} / p^{2}} \sum \delta_{m}^{\prime} \cos 4 m \phi \\
\tilde{\phi}= & \phi+1 / r^{2} \sum 4 m \delta_{m} \sin 4 m \phi
\end{aligned}
$$


where $\tilde{\theta}$ and $\Phi$ are the spherical angles made by the interface normal. is $\partial / \partial r$ and $\phi$ is the interface parameter chosen as the azimuthal angle of the original axisymmetric solution. Similarly,

$$
\begin{aligned}
\nabla^{2} z=-2 / p+\sum \delta_{m}^{\prime \prime} \cos 4 m \phi & +1 / r \sum \delta_{m}^{\prime} \cos 4 m \phi \\
& -\sum 16 m^{2} / r^{2} \delta_{m} \cos 4 m \phi .
\end{aligned}
$$

From these expressions substitution into equation (10) determines the linear Gibbs-Thomson shift.

It is useful to define the angle $\theta_{0}$ as $\tan ^{-1}(r / p)$. We group all the terms making up $\Delta_{\mu}$ into five distinct pieces

$$
-\Delta_{\mu}=A g_{1}+B g_{2}+C_{s} g_{3}+C_{c} g_{4}+D g_{5}
$$

where

$$
\begin{aligned}
& A=\cos ^{2} \theta_{0} \sum \frac{\delta_{m}^{\prime}}{p} \cos 4 m \phi \\
& B=\cos ^{2} \theta_{0} \sum \delta_{m}^{\prime \prime} \cos 4 m \phi \\
& C_{s}=\cot ^{2} \theta_{0} \sum \frac{4 m \delta_{m}}{p^{2}} \sin 4 m \phi \\
& C_{\mathrm{c}}=\cot ^{2} \theta_{0} \sum \frac{16 m^{2} \delta_{m}}{p^{2}} \cos 4 m \phi \\
& D=\cot ^{2} \theta_{0} \sum \frac{4 m \delta_{m}^{\prime}}{p^{2}} \sin 4 m \phi .
\end{aligned}
$$

Then, the coefficients are given explicitly as

$$
\begin{aligned}
& g_{1}=\cos \theta_{0} \cot \theta_{0}\left(2-3 \cos ^{2} \theta_{0}\right) \gamma \\
& +\left(3 \sin \theta_{0} \cos ^{2} \theta_{0}-\cos \theta_{0} \cot \theta_{0}\right) \frac{\partial^{2} y}{\partial \theta^{2}} \\
& -\cos ^{3} \theta_{0}\left(\frac{\partial \gamma}{\partial \ddot{\theta}}+\frac{\partial^{3} \gamma}{\partial \tilde{\theta}^{3}}\right)+\frac{\cot ^{2} \theta_{0}}{\sin \theta_{0}} \frac{\partial^{2} \gamma}{\partial \tilde{\phi}^{2}} \\
& -\frac{\cot \theta_{0}}{\sin \theta_{0}} \frac{\hat{c}^{3} \gamma}{\partial \bar{\theta} \partial \bar{\phi}^{2}} \\
& g_{2}=-\cos \theta_{0}\left(\gamma+\frac{\hat{c}^{2} \eta}{\partial \vec{\theta}^{2}}\right) \\
& \boldsymbol{g}_{3}=\cos ^{3} \theta_{0} \frac{\partial^{3} \tilde{y}^{\prime}}{\partial \tilde{\theta}^{2} \partial \tilde{\phi}}-\cos \theta_{0} \cot \theta_{0} \frac{\partial^{2} y}{\partial \tilde{\theta} \partial \tilde{\phi}} \\
& +\frac{\cot \theta_{0}}{\sin \theta_{0}} \frac{\hat{c}^{3} \gamma}{\partial \tilde{\phi}^{3}}+\left(2 \cos \theta_{0} \cot ^{2} \theta_{0}\right. \\
& \left.+\cos \theta_{0}+\cos ^{3} \theta_{0}\right) \frac{\partial_{y}}{\partial \tilde{\phi}} \\
& g_{4}=\cos \theta_{0} \cot \theta_{0} \frac{\partial_{\gamma}^{\gamma}}{\partial \hat{\theta}}+\cos \theta_{0} \gamma+\frac{\cot \theta_{0}}{\sin \theta_{0}} \frac{\partial^{2} \gamma}{\partial \tilde{\phi}^{2}} \\
& g_{5}=2 \cos \theta_{0} \frac{\hat{c}^{2} \gamma}{\partial \dot{\theta} \partial \hat{\phi}}-2 \cos \theta_{0} \cot \theta_{0} \frac{\partial y}{\partial \hat{\phi}} \text {. }
\end{aligned}
$$

The derivatives of $y$ can be evaluated using the explicit expression given in (2). This completes the evaluation of $\Delta$

Finally, it is easy to see how to put this result into the block matrix form needed in the main body of the text. Terms involving $A, B$ and $C_{\mathrm{c}}$ are proportional to $\cos 4 m \phi$; the corresponding coefficient functions have pieces independent of $\phi$ and pieces proportional to $\cos 4 \phi$. The resulting multiplications contains terms either still proportional to $\cos 4 m \phi$ or to

$\cos 4 m \phi \cos 4 \phi=1 / 2[\cos 4(m+1) \phi+\cos 4(m-1) \phi]$.

In this manner the surface energy couples different blocks of the matrix. The same thing happens for the other terms with the cosines replaced by sines, yielding terms

$\sin 4 m \phi \sin 4 \phi=-1 / 2[\cos 4(m+1) \phi-\cos 4(m-1) \phi]$.
The only singularities involve the $A$ terms, and these are handled by replacing $\delta_{m}^{\prime}$ with $\delta_{m}^{\prime}(r)-\delta_{m}^{\prime}(0)$, as described in the text.

\section{APPENDIX C}

In this Appendix we demonstrate the fact used in Section 5 that a linear shift in $z(r, \phi)$ of the form $\delta(r, \phi)=r^{m} \cos m \phi$ does not contribute to the integral on the right hand side of the steady-state shape selection equation. Another of stating this result is that the Ivantsov solution has an infinite number of zero modes of this form. This result was crucial in providing enough degrees of freedom to ensure that $\hat{c} z / \hat{c} r$ could be set equal to zero at the tip for general nonaxisymmetric $z$.

Before starting our derivation, we would like to note that this result must be true for $m=0,1$ by translation invariance and for $m=2$ by the existence of the Horvay-Cahn generalization of the Ivantsoy paraboloid of revolution to more general ellipsoidal paraboloids. We have not discovered any deep reason why this continues to be true for higher $m$ in the linear approximation, but it is true non" etheless. In the $m=0,1$ cases, the result is clearly valid to all orders in $\delta$; for $m=2$, higher orders in $\delta$ can always be absorbed by changing the undercooling. We have not yet investigated if there is any generalization of these nonlinear effects to higher $m$.

Let us start with the integral operator in the form given in equation (3) specialized to steady-state motion at velocity $v$. Up to an irrelevant constant, this can be expressed as

$$
\begin{aligned}
& \int \frac{v \mathrm{~d} x^{\prime} \mathrm{d} y^{\prime} \mathrm{d} \tau}{\tau^{3 / 2}} \\
& +\exp \left[\frac{-\left[\left(x-x^{\prime}\right)^{2}+\left(y-y^{\prime}\right)^{2}+\left(z-z^{\prime}-\tau \tau\right)^{\prime}\right]}{4 D \tau}\right] .
\end{aligned}
$$

We now set $z(x, y)=-\left(x^{2}+y^{2}\right) / 2 \rho+\delta(x, y)$ and expand. With the usual definition of $p=p v / 2 D$ and with rescaling lengths and times by $2 p$ and $p^{2}$ respectively, we find

$$
p \int \frac{\mathrm{d} x^{\prime} \mathrm{d} y^{\prime} \mathrm{d} \tau}{\tau^{3 / 2}} \times \exp \left[\frac{\left.+\left(x^{2}+y^{\prime 2}-x^{2}-y^{2}+p \tau\right)^{2}\right]}{\tau}\right]
$$

Following Pclce and Pomeau [16], we shift the integration variable $x^{\prime}, y^{\prime}$ by $x, y^{\prime}$ respectively and then rescale then by $\sqrt{t}$. This leads to

$$
\begin{aligned}
p \int \mathrm{d} x^{\prime} \mathrm{d} y^{\prime} \frac{\mathrm{d} \tau}{\tau} \mathrm{e}^{-\left(x^{\prime}+y^{\prime}\right]} \\
\quad \times \exp \left\{-\left[\left(x^{\prime 2}+y^{\prime 2}+p\right) \sqrt{\tau}\right]+2 x x^{\prime}+2 y y^{\prime}\right)^{\prime} \\
\quad \times\left[\left(x^{\prime 2}+y^{\prime 2}+p\right) \sqrt{\tau}+2 x x^{\prime}\right. \\
\left.\quad+2 y y^{\prime}\right] A\left(x, x^{\prime}, y, y^{\prime}, \tau\right)
\end{aligned}
$$

where $A$ is just the difference in the shift vectors $\delta$ after all the changes in variables. The final transformation defines $R=\left(x^{\prime 2}+y^{\prime 2}+p\right) \sqrt{t}$. The expression then becomes

$$
\begin{aligned}
2 p \int \mathrm{d} x^{\prime} \mathrm{d} y^{\prime} & \int_{0}^{\infty} \frac{\mathrm{d} T}{T} \mathrm{e}^{-\left(x^{\prime 2}+y^{\prime 2}\right)} \mathrm{e}^{-\left(\mathrm{T}+2 x x^{\prime}+2 y y^{\prime} z\right.} \\
& \times\left(T+2 x x^{\prime}+2 y y^{\prime}\right) \tilde{A}\left(x, x^{\prime}, y^{\prime}, y^{\prime}, T\right)
\end{aligned}
$$

with $\bar{A}$ derivable in the obvious manner from $A$. Note that the integral over $T$ can be extended to the range $-\infty \leqslant T \leqslant \infty$; any sign change in $T$ can be absorbed to a sign change in $x^{\prime}$ and $y^{\prime}$.

Let us focus on the structure of $\tilde{A}_{m}$ which arises from choosing the shift $\delta_{m}=r^{m} \cos m \phi=\operatorname{Re}\left(r e^{m \phi}\right)^{m}$. The various shifts and redefinitions combine to yield

$$
\tilde{A}_{m}=\operatorname{Re}\left[\left(\rho^{\prime} T e^{i \phi r}+r e^{i \phi}\right)^{m}-\left(r e^{i \phi}\right)^{m}\right]
$$

where

$$
\rho^{\prime} \equiv \frac{r^{\prime}}{r^{2}+p}
$$


The binomial expansion of this expression gives

$$
\sum_{k=1}^{m} b_{m, k} r^{m-k}\left(\rho^{\prime} T\right)^{k} \cos \left[k \phi^{\prime}+(m-k) \phi\right] .
$$

The final stage in the proof involves shifting $T$ in the integral by the factor $2 x x^{\prime}+2 y y^{\prime}$ resulting in a pure Gaussian exponent in $T$. This shift can equally well be written as $r r^{\prime} \cos \left(\phi-\phi^{\prime}\right)$. The integrals over $x^{\prime}, y^{\prime}$ can be rewritten in terms of an angular integral over $\phi^{\prime}$ and one over $r^{\prime}$. In this format, the factor multiplying the Gaussian in $T$ is

$$
\begin{aligned}
\sum_{k=1}^{m} b_{m, k} r^{m-k}\left(\rho^{\prime}\right)^{k}\left[T-r r^{\prime}\right. & \left.\cos \left(\phi-\phi^{\prime}\right)\right]^{k-1} \\
& \times \cos \left[k \phi^{\prime}+(m-k) \phi\right]
\end{aligned}
$$

where the original $1 / T$ has been used to reduce the power of the $T$ dependent piece in the above sum to $k-1$. Now imagine expanding the expression $\left[T-r r^{\prime} \cos \left(\phi-\phi^{\prime}\right)\right]^{k-1}$ in a binomial series. All the terms separately integrate to zero! This is true because they are being integrated versus $\cos \left[k \phi^{\prime}+(m-k) \phi\right]$ and different Fourier modes are orthogonal. This completes the required proof.

As stated above, it is not clear to us what happens to higher order in $\delta_{m}$ and therefore we do not know if this result can be extended to give additional solutions of the full non-linear zero surface tension problem. It is clear that the linear approximation must break down eventually as we move away from the tip because the shift vector grows at a faster rate that the underlying solution. This does not seem to affect the solvability mechanism, which in actuality is something that happens very close to the tip where the linearization is always valid.

\section{APPENDIX D}

In this Appendix, we review and extend the method developed in Ref. [9] for finding the asymptotic shape of the needle crystal in the presence of non-zero surface energy. It should be noted that the results quoted in that work for two dimensions are in fact incorrect; this was pointed out by Van Saarloos et al. in Ref. [36] and is due to the fact that the shift $\delta \sim 1 / r$ has a singularity near the tip which therefore becomes the most important region of integration on the right hand side of the shape equation. This is not a problem in three dimensions where $\delta$ grows at least as fast as $r$ and the tip region makes no significant contribution.

We start from the steady-state equation (4), first for the axisymmetric limit. As shown in [9], the leading term on the left hand side of the equation arises from the second piece of $\Delta_{\mu}^{(0)}$ in (18); asymptotically this term equals $\gamma_{0} / r$ and all other pieces are either $O\left(1 / r^{3}\right)$ or $O\left(\delta / r^{5}\right)$. To linear order in $\delta$, the right hand side has been derived in equation (15) as

$$
\begin{array}{r}
\int_{0}^{2 \pi} \frac{\mathrm{d} \phi}{2 \pi} \int_{0}^{\infty} r^{\prime} \mathrm{d} r^{\prime} \frac{\mathrm{e}^{z 0\left(r^{\prime}, \phi^{\prime}\right)-z(r, \phi)-d}}{d}\left[\delta\left(r^{\prime}\right)-\delta(r)\right] \\
\times\left[1-\frac{z_{0}\left(r^{\prime}\right)-z_{0}(r)}{d}\left(1+\frac{1}{d}\right)\right] .
\end{array}
$$

For large distances, the leading contribution to the integral comes from $r^{\prime} \leqslant r$. In this region, we can use the expansion

$$
d \simeq \frac{r^{2}-r^{\prime 2}}{2 p}+p\left(\frac{r^{2}+r^{\prime 2}-2 r r^{\prime} \cos \phi^{\prime}}{r^{2}-r^{\prime 2}}\right) .
$$

The angular integrals can all be done in terms of modified Bessel functions $I_{n}$. Changing variables to $q \equiv\left(r-r^{\prime}\right)$ $\left(r+r^{\prime}\right)$, we find the general result

$$
\begin{aligned}
& \frac{\delta(r)}{4 r^{2}} \int_{0}^{\infty} \frac{p^{2} \mathrm{~d} q}{q^{3}}\left(1-q^{2}\right) F_{m}(q) \\
& \quad \times\left\{I_{1}(z) \mathrm{e}^{-z} p\left(1-q^{2}\right)-I_{0}(z) \mathrm{e}^{-z}\left[2 q-p\left(1+q^{2}\right)\right]\right\}
\end{aligned}
$$

where $z=p\left(1-q^{2}\right) / 2 q$ and the function $F_{m}(q)$ is defined to be $1-\delta\left(\mathrm{r}^{\prime}\right) / \delta(\mathrm{r})$, for any power $\delta \sim r^{m}$; this can clearly be re-expressed as a function of $q$ via $r^{\prime} / r=1-q /(1+q)$.

From this last expression, it is clear that the generic behavior of the integral operator is to reduce by 2 the power of $r$ appearing in the shift $\delta$. To equate both sides of the equation, we therefore need to set $\delta \sim r$. Since there is no $1 / r^{2}$ term on the left hand side, the next door in $\delta$ would be $1 / r$ and case be neglected as fast as the linear version is concerned. If one is interested in the non-linear axisymmetric theory, there is a term of this type induced to second order in $\delta$ and therefore the next correction to linear is $\log r$. This has been discussed in [9] and will not be repeated here.

Our main interest is in extending these computations to the two mode approach of Section 5 . We have already seen in Appendix $C$ that $\delta(r)=r^{4} \cos 4 \phi$ does not contribute to the integral on the right hand side. So, our asymptotic analysis starts from the assumption that

$$
\delta_{1}^{A}(r)=f_{1} r^{4}+O\left(r^{3}\right) .
$$

The leading term on the left hand side can be found from the calculations in Appendix B. The leading term occurs in $g_{4} C_{C}$, which asymptotically equals $4(4+3 \epsilon) p \gamma_{0} f_{1} r \cos 4 \phi$. Note that the other terms formally of this order

$$
\begin{aligned}
\cos \theta_{0}\left[C_{\mathrm{s}} \frac{\partial \gamma}{\partial \widetilde{\phi}}+C_{\mathrm{c}}(\gamma\right. & -\gamma(\phi=0)) \\
& \left.+\frac{1}{\sin \theta_{0}}\left(C_{\mathrm{s}} \frac{\partial^{3} \gamma}{\partial \tilde{\phi}^{3}}+C_{\mathrm{c}} \frac{\partial^{2} \gamma}{\partial \tilde{\phi}^{2}}\right)\right]
\end{aligned}
$$

cancel to zero; this means that there is no axisymmetric piece to this order in $r$.

The linear term in $r$ must be cancelled by suitable choice of the cubic term in $\delta_{1}$, which will generate a linear term upon substitution into the integral. To find this coefficient, we need to generalize in (D3) to non-axisymmetric $\delta$. Following the same reasoning, we can derive the general formula for the integral over the shift $\delta(r, \phi)=r^{n} \cos 4 m \phi$ as $A_{n, m} r^{n-2} \cos 4 m \phi$ with the coefficients given by

$$
\begin{aligned}
\int_{0}^{\infty} \frac{\left(p^{2} \mathrm{~d} q\right.}{4 q^{3}}\left(1-q^{2}\right) e^{-z}\left\{p ( 1 - q ^ { 2 } ) \left[I_{1}(z)\right.\right. \\
\left.-\left(\frac{1-q}{1+q}\right)^{n} 1 / 2\left[I_{m+1}(z)+I_{m-1}(z)\right]\right] \\
\left.-\left[2 q-p\left(1+q^{2}\right)\right]\left[I_{0}(z)-\left(\frac{1-q}{1+q}\right)^{n} I_{m}(z)\right]\right\} .
\end{aligned}
$$

These integrals can all be evaluated numerically as functions of $p$. This can be used to cross check the requirement that $A_{4 m, m}=0$.

Using the above formula, we can continue the process of computing the asymptotic shape. The first axisymmetric term is again linear in $r$, now arising both from $\Delta_{\mu}^{(1)}$ and $f_{1}$. $\delta_{1}^{A}$ has terms of all orders below quartic. These results can then be used for the numerical analysis as described in the text. 